\title{
Estimation of Linear Stochastic Systems Over a Queueing Network
}

\author{
Michael Epstein, Abhishek Tiwari, Ling Shi and Richard M. Murray \\ Control and Dynamical Systems, California Institute of Technology \\ 1200 East California Blvd., Mail Code 104-44, Pasadena, CA 91125 \\ Email:\{epstein,atiwari,shiling,murray\}@cds.caltech.edu
}

\begin{abstract}
In this paper, we consider the standard state estimation problem over a congested packet-based network. The network is modeled as a queue with a single server processing the packets. This provides a framework to consider the effect of packet drops, packet delays and bursty losses on state estimation. We use a modified Kalman Filter with buffer to cope with delayed packets. We analyze the stability of the estimates with varying buffer length and queue size. We use high order Markov chains for our analysis. Simulation examples are presented to illustrate the theory.
\end{abstract}

\section{INTRODUCTION}

Traditionally the areas of control and communication networks are studied separately as they have almost distinctly different underlying assumptions. For example, control engineers generally assume perfect communication within the closed loop and data processing is done with zero time delay. On the other hand, in communication networks, data packets that carry the information can be dropped, delayed or even reordered due to the network traffic conditions. These different assumptions have for a long time inhibited researchers from the two fields from collaborating with each other. However, as new applications keep emerging, the two fields are coming closer together. For instance, advances in large scale integration and microelectromechanical system technology have made sensor networks an interesting area of research. In sensor networks, the measurement data from different sensors is sent to the controller through a data network where data packets might be dropped if the network has severe traffic.

In recent years, networked control problems have gained much interest. In particular, the state estimation problem over a network has been widely studied. The problem of state estimation and stabilization of a Linear Time Invariant (LTI) system with a finite bandwidth digital communication channel capacity was introduced by Wong and Brockett [12], [13] and further pursued by [8], [4], [10], [6]. In [2], Sinopoli et. al have discussed how packet loss can affect stable state estimation and they showed that there exists a certain threshold of the packet loss rate, above which the expected value of the error covariance becomes unbounded. They also provided lower and upper bounds of the threshold value. Following the spirit of [2], in [7], Liu and Goldsmith extended the above idea to the case where there are multiple sensors and the packets arrived from different sensors are dropped independently. They gave similar bounds on the

The authors are all equally contributed to this work packet loss rate for a stable estimation. The authors here gave a different point of view on the state estimation problem over general networks in [9]. There they showed that for any nonzero information gain, the state estimate is stable almost surely though the expected value of the error covariance could diverge.

In spite of the progress that the previous researchers have made, the problems they have studied have certain limitations. For example, in both [2] and [7], they assumed that packets are dropped independently, i.e. they model packet arrivals as i.i.d Bernoulli random variables which is certainly not true in the case of bursty communications or in queuing networks where adjacent packets are not dropped independently. The authors also did not consider packet delays or packets arriving out of order, both inherent with the communication network. In this paper we propose a queuing network model which is simple for analysis yet provides enough richness that it allows us to consider packet drops, delays and reordering in one setting. In the current work, we focus on packet drops and delays induced by the dedicated queue, as packet reordering is normally considered to be a rare event and is largely ignored by network community [11]. Even though packet reordering is rare, we plan to consider related issues in future work. It turns out that considering only the state estimation, packet delays have no significant effect by properly using the delayed packets. We propose an algorithm to modify the standard Kalman filter and hence take the delayed packets into account. Because of particular network structure we propose, the previous i.i.d packet drops [2] and [7] can be just considered as a special case of ours for some special network parameters.

The rest of the paper is organized as follows. In Section II, the mathematical model of our problem is given. We then study the state estimation problem over the proposed network and give conditions for the expected value of the error covariance to diverge in Section III. Section IV presents a method for analyzing the stability conditions, aided by several examples. We conclude with a short summary and overview on the future work in Section V.

\section{Problem SET UP}

Consider the following discrete-time LTI system

$$
\begin{aligned}
x_{k+1} & =A x_{k}+w_{k} \\
y_{k} & =C x_{k}+v_{k} .
\end{aligned}
$$


We assume $A$ is unstable, the pair $(A, C)$ is detectable, $x_{k} \in \mathbb{R}^{n}$ is the state vector, $y_{k} \in \mathbb{R}^{m}$ is the observation vector, $w_{k} \in \mathbb{R}^{n}$ and $v_{k} \in \mathbb{R}^{m}$ are Gaussian random vectors with zero mean and covariance matrices $Q \geq 0$ and $R>0$, respectively. Assume $w_{s}$ is independent from $w_{k}$ and $v_{s}$ is independent from $v_{k}$ for $s \neq k$, and $w_{s}$ is also independent from $v_{k}$ for all $s$ and $k$.

The problem of interest to us is to get a stable estimate of the state vector $x_{k}$ at time $k$ given all past measurement $y_{0}, \ldots, y_{k}$ sent over a packet based network. Each packet $y_{i}, i \in\{0, \ldots, k\}$ can be dropped or delayed by the network. The packet-based network is modeled as follows (see Fig. 1). The observation unit (sensor) is directly linked to a router, which drops packets based on the network traffic condition. If the queue is full, the router drops each arriving packet with probability one. As the queue has more space, the arriving packets are dropped with relatively lower probability. We will refer to the function describing the packet acceptance probability w.r.t. the queue length as $\lambda=\mathbb{F}(Q)$.

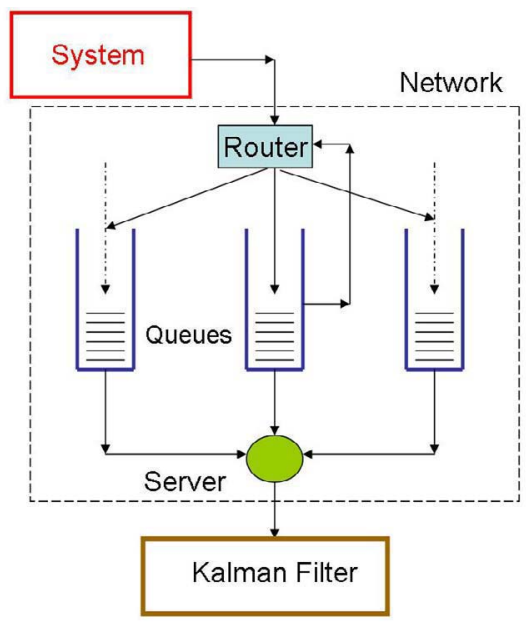

Fig. 1. A simple queueing network model

Ideally the router may also route the packets to other queues, thus resulting in packets being received out of order at the Kalman filter. Since packet reordering is not so critical in network estimation problems, we assume that all the packets are routed to the same queue. The queue is assumed to have a finite capacity of $N>0$ packets. There is a time shared server which processes a packet from the queue with probability $\rho$ at any given time instant. Once a packet is processed, it is delivered to a Kalman filter. The Kalman filter is used to estimate the state vector.

To take delayed packets into account we propose an algorithm that uses a Kalman filter with buffer, see table I. The Kalman filter always performs the time update (prediction) step. The measurement update (correction) step is performed on the estimate of $x_{k}$ only if the packet containing the information $y_{k}$ is eventually received (delayed or instantly). See Table I for a description of the algorithm and [1] for a detailed description of the time and measurement update steps. Let $L$ be the size of the buffer. Therefore any packet
TABLE I

ALGORITHM FOR MODIFIED KALMAN FILTER WITH BUFFER

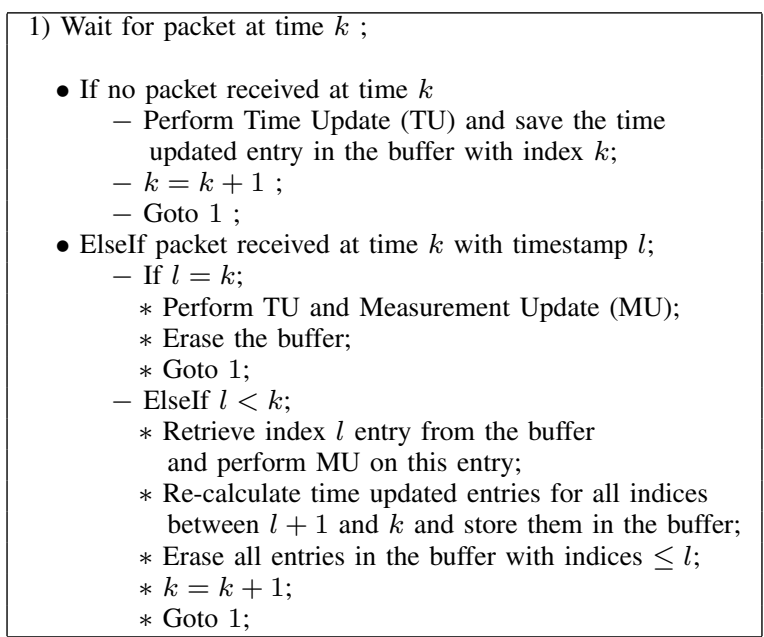

which is delayed more than $L$ time steps can not be used by the Kalman filter. We assume all packets to be timestamped. Let the timestamp on the packet received at time $k$ be $l$.

If the network has the information that any packet older than $L$ time steps waiting in the queue is useless, then it can flush all such packets. This assumption of flushing of old enough packets in the queue simplifies the analysis a great deal and will enable us to employ $L$ th order Markov chains to analyze such queueing networks in Section III.

Since the Kalman filter has a finite buffer length $L$, it outputs the best estimate of $x_{k}$ at time $k+L$. If the packet $y_{k}$ is delayed by more than $L$ steps then it will be flushed at the queue. Therefore in this paper our metric of estimation will be the value of the error covariance matrix $P_{k}^{k+L}=$ $\mathbb{E}\left(e_{k}^{k+L} e_{k}^{k+L}\right)$, where $e_{k}^{k+L}=x_{k}-\hat{x}_{k}^{k+L}$ and the superscript indicates that we look at this metric at time $k+L$, after which the estimate is fixed and cannot be improved further. For notational simplicity we will drop the superscript $k+L$ for the rest of this paper.

For our analysis let us define the indicator function

$$
I_{k}= \begin{cases}1 & k^{t h} \text { packet received instantly or with delay } \\ 0 & k^{t h} \text { packet dropped or flushed }\end{cases}
$$

and the following relations for $X \in \mathbb{S}_{n}$, the set of all positive semi-definite matrices of size $n$.

$$
\begin{aligned}
h(X) & \triangleq A X A^{\prime}+Q \\
f(X) & \triangleq A X C^{\prime}\left(C X C^{\prime}+R\right)^{-1} C X A^{\prime} \\
g(X) & \triangleq h(X)-f(X)
\end{aligned}
$$

At this point we would like to remind the reader that under the estimation scheme described in Table I the recursion of the error covariance matrix can be written in terms of $h$ and $g$ as,

$$
P_{k+1}= \begin{cases}h\left(P_{k}\right) & I_{k+1}=0 \\ g\left(P_{k}\right) & I_{k+1}=1\end{cases}
$$


The problem at hand is to determine conditions on the function $\mathbb{F}$ at the router and the server service probability $\rho$, for which the expected value of the error covariance remains bounded as $k \rightarrow \infty$. In the following section we establish that as long as the staying probability in the dropped or flushed state is below certain threshold the estimates are stable. We find this staying probability in terms of $\mathbb{F}$ and $\rho$ for three different combinations of $L$ (buffer length) and $N$ (queue length).

\section{Stability of The ERror Covariance}

Before we state our main result we derive and present some properties of the functions $h$ and $g$.

Lemma 1: If $X \geq Y$, then $g(X) \geq g(Y)$ and $h(X) \geq$ $h(Y)$.

Proof: See [2].

QED

Lemma 2: If $U \in \mathbb{S}_{n}^{+}$, the set of all positive definite matrices of size $n$, and $V \in \mathbb{S}_{n}$, then $\exists$ a scalar $t \geq 0$ such that $t U-V \in \mathbb{S}_{n}$.

Proof: By Weyl's Theorem [5], $t \geq 0$

$$
\begin{aligned}
\lambda_{\min }(t U-V) & \geq \lambda_{\min }(t U)+\lambda_{\min }(-V), \\
& =t \lambda_{\min }(U)-\lambda_{\max }(V),
\end{aligned}
$$

where $\lambda_{\min }$ is the minimum eigenvalue and $\lambda_{\max }$ is the maximum eigenvalue So any $t \geq \frac{\lambda_{\max }(V)}{\lambda_{\min }(U)}$ proves the Lemma. Such a $t$ always exists because $\lambda_{\min }(U)>0$.

QED

Lemma 3: $g(X) \geq Q, \forall X \geq 0$ and if $C$ is invertible then, $g(X) \leq A C^{-1} R C^{-1} A^{\prime}+Q, \forall X \geq 0$.

Proof: Clearly $g(X) \geq g(0)=Q$. For any $X \geq 0$, as $C^{-1} R C^{\prime-1} \in \mathbb{S}_{n}^{+}$, by Lemma 2 , there exists $t \geq 0$ such that

$$
\begin{aligned}
X & \leq t C^{-1} R C^{\prime-1} \\
g(X) & \leq g\left(t C^{-1} R C^{\prime-1}\right), \\
& =t /(t+1) A C^{-1} R C^{\prime-1} A^{\prime}+Q, \\
& \leq A C^{-1} R C^{\prime-1} A^{\prime}+Q,
\end{aligned}
$$

by using Lemma 1 in $a$.

QED

Lemma 4: (a) If $\mathrm{A}$ is unstable then

$$
\lim _{k \rightarrow \infty} h^{k}\left(X_{0}\right)=\infty, \forall X_{0} \in \mathbb{S}_{n}
$$

(b) If the spectral radius of $A, \alpha<1$ and the pair $(A, \sqrt{Q})$ is observable, then the Lyapunov difference equation $X_{k+1}=h\left(X_{k}\right)$ converges to a unique positive semidefinite solution $T>0$ as $k \rightarrow \infty$. In other words the following infinite sum

$$
\lim _{k \rightarrow \infty}\left[A^{k} X_{0} A^{\prime k}+\sum_{m=0}^{k-1} A^{m} Q A^{\prime m}\right]
$$

is a finite positive definite matrix $T>0$ for all $X_{0} \geq 0$, where $T=h(T)$.
Proof: See [3]

QED

Under the flushing assumption introduced in Section II, the fate of the $k$ th packet does not depend on what happened to packets with timestamps $k-L-1$ or less, and thus we can analyze the network using an $L$ th order Markov chain. We first present stability results for $L=1$ and then later we generalize them for all $L$.

Define the following probabilities

$$
\begin{aligned}
\omega_{h \rightarrow h} & =\operatorname{Pr}\left[I_{k+1}=0 \mid I_{k}=0\right] \\
\omega_{h \rightarrow g} & =\operatorname{Pr}\left[I_{k+1}=1 \mid I_{k}=0\right] \\
\omega_{g \rightarrow g} & =\operatorname{Pr}\left[I_{k+1}=1 \mid I_{k}=1\right] \\
\omega_{g \rightarrow h} & =\operatorname{Pr}\left[I_{k+1}=0 \mid I_{k}=1\right]
\end{aligned}
$$

Let $\eta_{g}$ be the steady state probability of the event $I_{k}=1$, i.e. the $k$ th packet is eventually received. For technical reasons lets assume that first packet is received with probability $\eta_{g}$. In other words the Markov chain describing the packet drop process starts at steady state. This assumption here is just for the simplicity of the proofs.

Theorem 1: For a Kalman filter with buffer length $L=1$, the estimates are stable/unstable under the following conditions.

(a) Let $(A, C)$ be detectable and $(A, \sqrt{Q})$ be observable, now if

$$
\omega_{h \rightarrow h} \geq \frac{1}{\alpha^{2}},
$$

where $\alpha$ is the spectral radius of $A$, then $\lim _{k \rightarrow \infty} \mathbb{E}\left(P_{k}\right)$ is unbounded.

(b) If $C$ is invertible and if

$$
\omega_{h \rightarrow h}<\frac{1}{\alpha^{2}},
$$

then $\lim _{k \rightarrow \infty} \mathbb{E}\left(P_{k}\right)$ is bounded for all initial conditions $P_{0} \in \mathbb{S}_{n}$.

Proof:

(a) $P_{k+1}$ can take $2^{k+1}$ different values with different probabilities for a given value of $P_{0}$ depending on the values of $I_{1}, I_{2} \cdots I_{k+1}$. From Lemma 3 we know that $g(X) \geq Q$, and from Lemma 1 we know that $h$ is an increasing function. Therefore

$$
\begin{aligned}
\mathbb{E}\left[P_{k}\right] & \geq \eta_{g} Q+\frac{\left(1-\eta_{g}\right)}{\omega_{h \rightarrow h}} \omega_{h \rightarrow h}^{k} h^{k}\left(P_{0}\right) \\
& +\frac{\eta_{g} \omega_{g \rightarrow h}}{\omega_{h \rightarrow h}} \sum_{i=0}^{k-2} \omega_{h \rightarrow h}^{i+1} h^{i+1}(Q)
\end{aligned}
$$

To illustrate how we obtain the above inequality we consider the case when $k=3$, in Table II. The right hand side of the above equation is the inner product of the 1 st and 3rd rows of the table. Using Lemma 4 $\lim _{k \rightarrow \infty} \mathbb{E}\left(P_{k}\right)$ is unbounded if

$$
\omega_{h \rightarrow h} \geq \frac{1}{\alpha^{2}}
$$


TABLE II

ILLUSTRATION OF HOW TO FIND THE LOWER BOUND

\begin{tabular}{c|c|c} 
Probabilities & Values & Lower bounds \\
\hline$\left(1-\eta_{g}\right) \omega_{h \rightarrow h}^{2}$ & $h^{3}\left(P_{0}\right)$ & $h^{3}\left(P_{0}\right)$ \\
$\eta_{g} \omega_{g \rightarrow h} \omega_{h \rightarrow h}$ & $h^{2} g\left(P_{0}\right)$ & $h^{2}(Q)$ \\
$\left(1-\eta_{g}\right) \omega_{h \rightarrow g} \omega_{g \rightarrow h}$ & $h g h\left(P_{0}\right)$ & $h(Q)$ \\
$\eta_{g} \omega_{g \rightarrow g} \omega_{g \rightarrow h}$ & $h g^{2}\left(P_{0}\right)$ & $h(Q)$ \\
$\left(1-\eta_{g}\right) \omega_{h \rightarrow h} \omega_{h \rightarrow g}$ & $g h^{2}\left(P_{0}\right)$ & $Q$ \\
$\eta_{g} \omega_{g \rightarrow h} \omega_{h \rightarrow g}$ & $g h g\left(P_{0}\right)$ & $Q$ \\
$\left(1-\eta_{g}\right) \omega_{h \rightarrow g} \omega_{g \rightarrow g}$ & $g^{2} h\left(P_{0}\right)$ & $Q$ \\
$\eta_{g} \omega_{g \rightarrow g}^{2}$ & $g^{3}\left(P_{0}\right)$ & $Q$ \\
\hline
\end{tabular}

(b) If $C$ is invertible then we can find an upper bound using Lemma 3

$$
\begin{aligned}
\mathbb{E}\left[P_{k}\right] & \leq \eta_{g} M+\frac{\left(1-\eta_{g}\right)}{\omega_{h \rightarrow h}} \omega_{h \rightarrow h}^{k} h^{k}\left(P_{0}\right) \\
& +\frac{\eta_{g} \omega_{g \rightarrow h}}{\omega_{h \rightarrow h}} \sum_{i=0}^{k-2} \omega_{h \rightarrow h}^{i+1} h^{i+1}(M)
\end{aligned}
$$

where $M=A C^{-1} R C^{\prime-1} A^{\prime}+Q$ Now the first term on the right hand side is finite. From Lemma 4 the second term is finite as $k \rightarrow \infty$ if $\omega_{h \rightarrow h} \alpha^{2}<1$. The third term after summing the geometric series can be rewritten as

$$
\begin{gathered}
\frac{\eta_{g} \omega_{g \rightarrow h}}{\omega_{h \rightarrow h}}\left[\sum_{i=1}^{k-1} \tilde{A}^{i} M\left(\tilde{A}^{i}\right)^{\prime}\right. \\
\left.+\frac{\omega_{h \rightarrow h}}{1-\omega_{h \rightarrow h}} \sum_{i=0}^{k-2} \tilde{A}^{i} Q\left(\tilde{A}^{i}\right)^{\prime}\left(1-\omega_{h \rightarrow h}^{k-1-i}\right)\right]
\end{gathered}
$$

where $\tilde{A}=\sqrt{\omega_{h \rightarrow h}} A$. Again using Lemma 4 we know that this term is finite as $k \rightarrow \infty$ if $\omega_{h \rightarrow h} \alpha^{2}<1$.

QED

Now we state a general result for buffer length $L$ but before that we define

$$
\omega_{\text {Ltimes }}^{h h \cdots h} \rightarrow h
$$

as the probability that the $k$ th packet is dropped or flushed given that the $L$ previous packets are dropped or flushed.

Corollary 1: For buffer length $L$

(a) Let $(A, C)$ be detectable and $(A, \sqrt{Q})$ be observable, now if

$$
\omega_{\underbrace{h h \cdots h}_{\text {Ltimes }} \rightarrow h} \geq \frac{1}{\alpha^{2}},
$$

where $\alpha$ is the spectral of $A$, then $\lim _{k \rightarrow \infty} \mathbb{E}\left(P_{k}\right)$ is unbounded.

(b) If $C$ is invertible and if

$$
\omega_{\text {Ltimes }}^{h h \cdots h \rightarrow h}<\frac{1}{\alpha^{2}},
$$

then $\lim _{k \rightarrow \infty} \mathbb{E}\left(P_{k}\right)$ is bounded for all initial conditions $P_{0} \in \mathbb{S}_{n}$.

Proof: The proof is very similar to that of Theorem 1 . We omit the detailed proof due to space constraints. As an example, the lower bound and upper bound on $\mathbb{E}\left(P_{k}\right)$ for $L=2$ are given by the relation

$$
\begin{gathered}
\eta_{g} \mathcal{X}+\eta_{g h} h(\mathcal{X})+\sum_{i=0}^{k-3} \eta_{g h} \omega_{g h \rightarrow h} \omega_{h h \rightarrow h}^{i} h^{i+2}(\mathcal{X}) \\
+\eta_{h h} \omega_{h h \rightarrow h}^{k-2} h^{k}\left(P_{0}\right)
\end{gathered}
$$

with $\mathcal{X}=Q$ and $\mathcal{X}=M$ for the lower and upper bound respectively. In this case the analysis tool is a second order Markov chain. Here $\eta_{g h}$ is the steady state probability that the $k-1$ th packet is eventually received and $k$ th packet is dropped or flushed. Similarly, $\eta_{h h}$ is the steady state probability that both $k-1$ th and $k$ th packets are dropped or flushed. While $\omega_{g h \rightarrow h}$ is the probability that $k+1$ th packet is dropped or flushed given that $k-1$ th was received and $k$ th lost.

\section{Analyzing Different Buffer ANd Queue LENGTH COMBINATIONS}

In this section we will analyze different combinations of buffer and queue length. We will make use of Markov chains to facilitate the analysis. For buffer length $L$, the state of the Markov chain at time $k$ will be given by $M_{k}=\left(M_{k}^{1}, M_{k}^{2}, \cdots, M_{k}^{L}\right)$, where $M_{k}^{i} \in$ $\{-1,0,1,2,3, \cdots, L, \infty\}$ represents what happens to the data packet sent to the router at time $k-i+L$. Note $M_{k}^{i}=M_{k-1}^{i+1}$ for $1 \leq i<L$. If $M_{k}^{i}=-1$, the packet is dropped by the router, i.e. does not enter the queue. If $M_{k}^{i} \geq 0$, it signifies the packet entered the queue and if $0 \leq M_{k}^{i}=m \leq L$ the packet is passed by the server to the Kalman filter with delay $m$ timesteps. If $M_{k}^{i}=\infty$ the packet entered the queue but was not processed by the server for $L$ timesteps and hence was flushed from the queue. As described in Corollary 1, we are interested in the staying probability that all packets do not get processed by the Kalman filter, that is they are either dropped by the router $\left(M_{k}^{i}=-1\right)$ or flushed from the queue $\left(M_{k}^{i}=\infty\right)$. Switching notation from here on to $f(\lambda, \rho)=\omega_{\underbrace{h h \cdots h}_{\text {Ltimes }} \rightarrow h}$,

we see

$$
\begin{aligned}
f(\lambda, \rho) & =\operatorname{Pr}\left[M_{k}^{i}=\{-1, \infty\}, \forall i=1, \cdots, L\right. \\
M_{k-j}^{i} & =\{-1, \infty\}, \forall i=1, \cdots, L \text { and } \forall j=1, \ldots, L]
\end{aligned}
$$

where as before $\lambda=\mathbb{F}(Q)$ a vector function of the probability the router will allow a packet to enter the queue based on the number of packets currently in the queue (i.e. $\lambda_{0}$ for an empty queue, $\lambda_{1}$ for 1 packet in the queue, etc.) and $\rho$ is the probability the server processes a packet a from the queue. We will denote the value of $f(\lambda, \rho)$ as $f_{b L q N}$ for buffer length $L$ and queue length $N$. Note the smaller the value of $f$ the better the estimate, in the sense that estimates of larger unstable system by the Kalman filter with buffer algorithm will not diverge. 


\section{A. Buffer Length $=1$, Queue Length $=1$}

Here we consider a Kalman filter with buffer length 1 and a network with queue that can hold only 1 packet. Packets are flushed from the queue if they are not processed by the server after 1 time step. This leads to four possibilities for any packet arriving at the router at time $k$; the packet is either (i) dropped at the router, (ii) serviced at time $k$, (iii) serviced at time $k+1$, or (iv) flushed from the que after time $k+1$. This is depicted by the Markov chain in Fig. 2 (for queue length 1 , set $\lambda_{1}=0$ ).

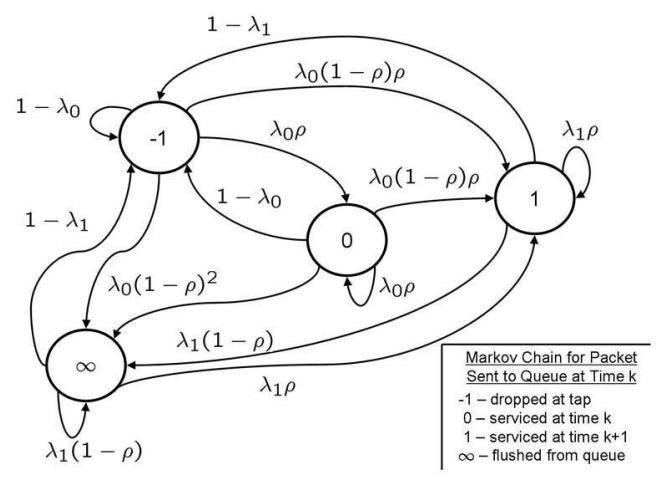

Fig. 2. Markov chain for buffer length 1 and queue length 1 or 2 .

Since the network is a first order Markov chain, according to Eqn. (13) we see

$$
\begin{aligned}
f_{b 1 q 1} & =\operatorname{Pr}\left[M_{k}^{1}=\{-1, \infty\} \mid M_{k-1}^{1}=\{-1, \infty\}\right] \\
& =\frac{\pi_{-1}\left(1-\lambda_{0}+\lambda_{0}(1-\rho)^{2}\right)+\pi_{\infty}}{\pi_{-1}+\pi_{\infty}},
\end{aligned}
$$

where $\pi_{i}$ represents the steady state probability of the Markov chain in Fig. 2 being in state $i$. These can be easily computed and hence

$$
f_{b 1 q 1}=\frac{-5 \lambda_{0} \rho+2 \lambda_{0}^{2} \rho^{2}-\lambda_{0}^{2} \rho^{3}+1+2 \lambda_{0} \rho^{2}+1}{-3 \lambda_{0} \rho+1+\lambda_{0}+\lambda_{0} \rho^{2}} .
$$

B. Buffer Length $=1$, Queue Length $=2$

Now the queue length is increased to 2, thereby ensuring the queue will never appear full to the router. Hence the server will allow packets into the queue with probability $\lambda_{1}$ when a packet is in the queue, as opposed to probability 0 when the queue length is 1 . The Markov chain describing this network is shown in Fig. 2. Note that setting $\lambda_{1}=0$ will revert to this system to be equivalent to that of queue length 1 .

The function for stability for this case and all the remaining cases in this paper can be readily computed but are too complicated to write out. To compare queue length 1 with queue length 2 one must look at the value of $\omega_{h \rightarrow h}$ for both cases. It is not too difficult to compute

$$
\begin{aligned}
& f_{b 1 q 2}-f_{b 1 q 1}= \\
& \lambda_{0} \lambda_{1} \rho\left(\lambda_{0}-1\right)(\rho-1)^{2}\left(\lambda_{0} \rho^{2}-2 \lambda_{0} \rho+1\right) / \\
& {\left[\left(1+\lambda_{0} \rho^{2}+\lambda_{0}-3 \lambda_{0} \rho\right)\right.} \\
& \left.\left(1+\lambda_{0} \lambda_{1} \rho+\lambda_{0} \rho^{2}+\lambda_{0}-3 \lambda_{0} \rho-\lambda_{1}\right)\right]
\end{aligned}
$$

which is negative for all $0 \leq \lambda_{0}, \lambda_{1}, \rho \leq 1$, meaning $f_{b 1 q 2} \leq$ $f_{b 1 q 1}$. Hence for fixed buffer length of 1 it is better to use queue length 2 .

\section{Buffer Length $=2$, Queue Length $=1$}

The next scenario considered is that of buffer length 2 and queue length 1 . In this case packets are flushed from the queue if they are not processed by the server after 2 time steps. There are five possibilities for any packet arriving at the router at time $k$; the packet is either (i) dropped at the router, (ii) serviced at time $k$, (iii) serviced at time $k+1$, (iv) serviced at time $k+2$, or (v) flushed from the que after time $k+2$. Since packets can stay in the queue for 2 time steps, this queueing network must be described by a second order Markov chain as depicted in Fig. 3. We can compare

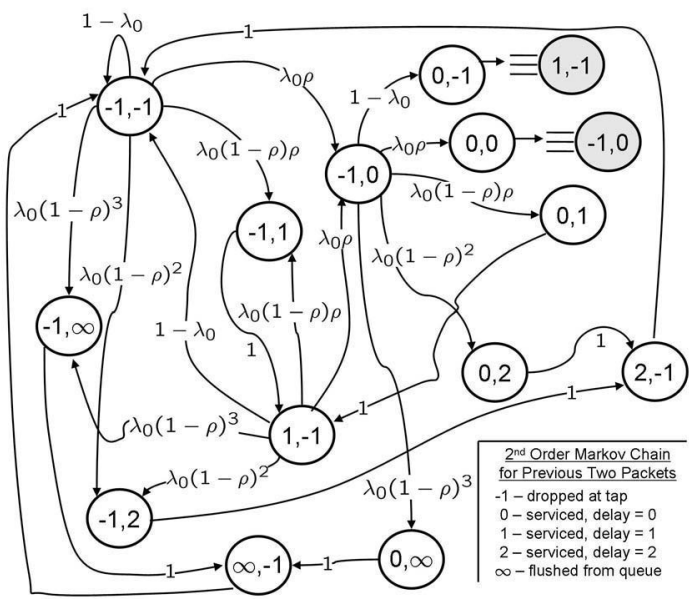

Fig. 3. Markov chain for buffer length 2 and network with queue length 1. The states of the Markov chain represent what happens to the last two packets sent to the queue. For clarity the shaded circles are used to show that the states entering these circles transition to the same states with the same probabilities as the unshaded circles with same number, i.e. state $(0,0)$ has the same transition probabilities to the same states as state $(-1,0)$.

the stability properties with that of the previous situations. It can be shown $f_{b 2 q 1} \leq f_{b 1 q 1}$ for all $0 \leq \lambda_{0}, \rho \leq 1$ so using a buffer length of 2 will improve performance when the queue length is fixed at 1.

\section{Buffer Length $=2$, Queue Length $=2$}

The queue length is increased to 2 while keeping the buffer length fixed at 1 . The resulting Markov chain is omitted for brevity, but it is very similar to that of Fig. 3 with the addition of the states $(1,1) ;(1,2) ;(1, \infty) ;(2,2) ;(2, \infty) ;(\infty, 2) ;(\infty, \infty)$ and appropriate changes to the state transition probabilities. Again it is possible to show $f_{b 2 q 2} \leq f_{b 2 q 1}$ for all $0 \leq \lambda_{0}, \lambda_{1}, \rho \leq 1$, and consequently $f_{b 2 q 2} \leq f_{b 1 q 1}$.

\section{E. Buffer Length $=2$, Queue Length $=3$}

The queue length is increased to 3 . Once again it can be shown $f_{b 2 q 3} \leq f_{b 2 q 2}$ for all $0 \leq \lambda_{0}, \lambda_{1}, \rho \leq 1$, and consequently $f_{b 2 q 3} \leq f_{b 2 q 1}$ and $f_{b 2 q 3} \leq f_{b 1 q 1}$. 


\section{F. Simulations}

We compare estimates using the Kalman filter with buffer algorithm for the buffer and queue length combinations listed above. A common initial condition for $P_{0}$ was used in all simulations. Randomly choosing a value from the steady state distribution of the Markov chains determined if the first $L$ packets were passed to the Kalman filter which then determined the number of packets of the queue for time $L+1$ (i.e. for buffer length 2 queue length 2 if the random toss resulted in choosing the Markov state $(-1,2)$ the first data packet would be dropped at the router and the second delivered to the Kalman filter with delay 2, resulting in the queue containing 1 packet after timestamp 2.). After time $L+1$ the router allows packets into the queue based on the number of packets currently in the queue and the probability vector $\lambda$, likewise the server will pass packets from the queue to the Kalman filter with probability $\rho$.

The network properties are given by $\left(\lambda_{0}, \lambda_{1}\right)=(0.5,0.5)$ and $\rho=0.6$. The first system is given by Eqn. (1) - (2) with $A=1.15, C=1, Q=20, R=2.5$. All the network combinations listed above will keep $\mathbb{E}\left[P_{k}\right]$ bounded. A total of 100,000 simulations were run for each buffer queue length combination with the same initial condition $P_{0}=200$. Plotted in Fig. 4 is the average across all the runs for each combination. The best estimates are by $b 2 q 2$ and $b 2 q 3$ which are virtually identical, followed by $b 2 q 1$ and $b 1 q 2$ which are also virtually identical, $b 1 q 1$ gives the worst estimate.

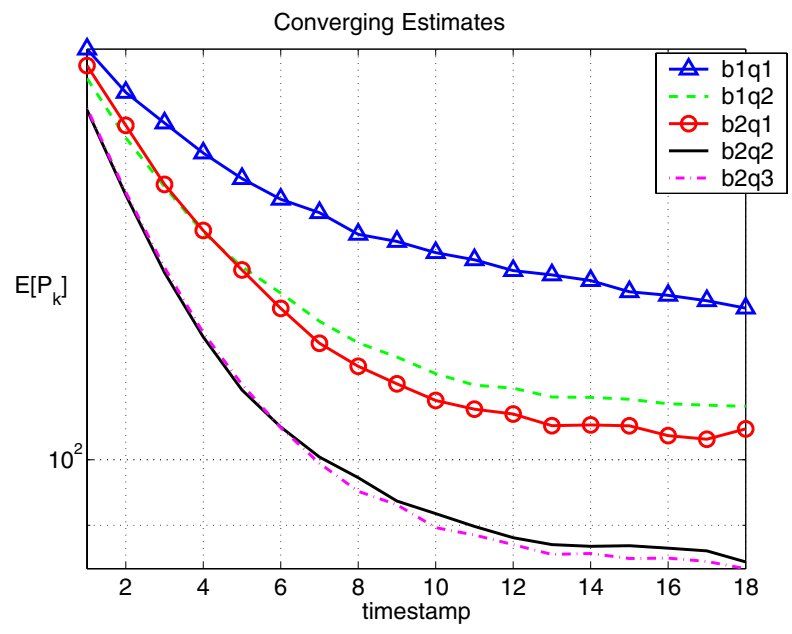

Fig. 4. Comparison of $\mathbb{E}\left[P_{k}\right]$ for different buffer and queue length combinations, all converge.

The value of $A$ was increased to 1.33 while holding all other parameters fixed. All of the estimates in this case will diverge as can be seen in Fig. 5. The relative quality of the estimates is the same as in the converging case.

\section{CONClusions AND Future Work}

In this paper we present both an algorithm for estimation over a queueing network that deals with dropped and delayed packets and stability conditions. The algorithm consists of a modified Kalman filter with buffer. We also give an

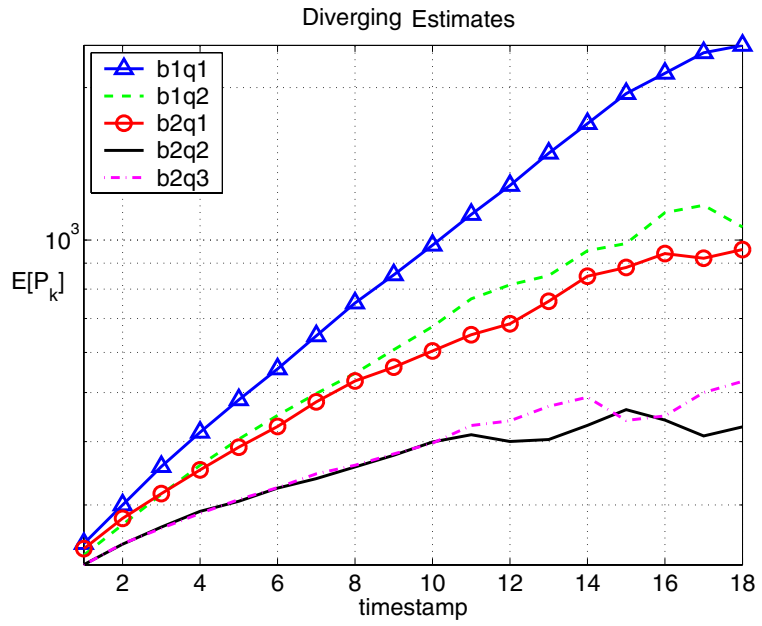

Fig. 5. Comparison of $\mathbb{E}\left[P_{k}\right]$ for different buffer and queue length combinations, all diverge.

analysis method for arbitrary buffer and queue length based on Markov chains whose order is determined by the buffer length. Simulations of several different queue and buffer lengths are presented.

There are several avenues of research to follow up on this paper. Namely, looking at other network congestion models. Designing an algorithm to handle packets that are received out of order. Joint synthesis of the network and estimation algorithm with a feedback controller.

\section{REFERENCES}

[1] B. D. O. Anderson and J. B. Moore. Optimal Filtering. Prentice-Hall, Inc., 1979.

[2] B.Sinopoli, L.Schenato, M.Franceschetti, K.Poolla, M.Jordan, and S.Sastry. Kalman filtering with intermittent observations. IEEE Transactions on Automatic Control, 49(9):1453-1464, 2004.

[3] Z. Gajić and M. Qureshi. Lyapunov Matrix Equation in System Stability and Control. Academic Press, 1995.

[4] G.N.Nair and R.J.Evans. Communication-limited stabilization of linear systems. In Proceedings of the 39th Conf. on Decision and Contr., volume 1, pages 1005-1010, Dec 2000.

[5] R. A. Horn and C. R. Johnson. Matrix Analysis. Cambridge University Press, 1985.

[6] I.R.Petersen and A.V.Savkin. Multi-rate stabilization of multivariable discrete-time linear systems via a limited capacity communication channel. In Proceedings of the 40th Conf. on Decision and Contr. volume 1, pages 304-309, Dec 2001.

[7] Xiangheng Liu and Andrea Goldsmith. Kalman filtering with partial observation losses. IEEE Control and Decision, 2004.

[8] R.W.Brockett and D.Liberzon. Quantized feedback stabilization of linear systems. IEEE Trans. Automat. Contr., 45, July 2000.

[9] Ling Shi, Michael Epstein, Ahbishek Tiwari, and Richard.M.Murray. Estimation with information loss: Asymptotic analysis and error bounds. CDC-ECC05, submitted, 2005.

[10] S. C. Tatikonda. Control Under Communication Constraints. PhD thesis, Massachusetts Institute of Technology, 2000.

[11] W.Stevens. Rfc 2001: Tcp slow start, congestion avoidance, fast retransmit, and fast recovery algorithms. Jan 1997.

[12] W.S.Wong and R.W.Brochett. Systems with finite communication bandwidth-part i: State estimation problems. IEEE Trans. Automat. Contr., 42, Sept 1997.

[13] W.S.Wong and R.W.Brochett. Systems with finite communication bandwidth-part ii: Stabilization with limited information feedback. IEEE Trans. Automat. Contr., 44, May 1999. 